\title{
Polímeros Condutores Intrínsecos e Seu Potencial em Blindagem de Radiações Eletromagnéticas
}

\author{
Roselena Faez, Mirabel C. Rezende \\ Divisão de Materiais, IAE, CTA, S. J osé dos Campos
}

Inácio M. Martin

Instituto de Física, UNICAMP

Marco-A. De Paoli Laboratório de Polímeros Condutores e Reciclagem, UNICAMP

Resumo: A procura por novos materiais que possuam propriedades magnéticas e dielétricas adequadas ao uso em absorvedores de radiação eletromagnética é de grande importância. Dentre os materiais em estudo, os polímeros condutores têm sido aplicados como centros de absorção de radiação, devido à possibilidade de variação da sua condutividade com a freqüência da radiação das ondas incidentes, sendo esta pesquisa o estado da arte no processamento de materiais absorvedores de radiação (MAR). O objetivo deste trabalho é apresentar a ampla faixa de propriedades dos polímeros condutores, correlacionando-as com o potencial de aplicação destes materiais como aditivos no processamento de MAR. Uma breve análise dos resultados obtidos com uma blenda de EPDM contendo 30-80\%(m/m) de PAni-DBSA e espessuras de 1 e $3 \mathrm{~mm}$, analisadas na faixa de 8-12 GHz, mostra que o material absorveu na faixa de 50 a $90 \%$ da radiação incidente.

Palavras-chave: Polímeros condutores, materiais absorvedores de radiação, propriedades dielétricas, propriedades magnéticas.

\section{Intrinsically Conducting Polymers and Their Potencial in Electromagnetic Interference Shielding}

Abstract: The search for new materials possessing magnetic and dieletric properties adequated to be used as radar absorbing materials has been of great importance. Among the materials under study, conducting polymers have been applied due to the possibility of variation of their conductivity with the frequency of the incident radiation. These research areas have been considered the state of the art in the processing of radar absorbing materials. The aim of this work is to present the large range of properties of the conducting polymers and their correlation with the potential application of these materials as electromagnetic radiation absorbers. Some results are also presented on blends of EPDM and 30-80\% (w/w) of PAni-DBSA with thickness of 1 and $3 \mathrm{~mm}$, analysed in the range of 8-12 GHz, which absorbed in the range of 50-90\% of incident radiation.

Keywords: Conducting polymers, radar absorbing materials, dieletrics and magnetic properties.

\section{Introdução}

Nos últimos 15 anos, os polímeros condutores têm sido estudados como aditivos com excelente potencial de aplicação no processamento de materiais utilizados nas áreas de interferência eletromagnética e de absorção de microondas, em substituição aos materiais absorvedores convencionais, que utilizam ferritas, partículas metálicas e de carbono. $\mathrm{O}$ maior interesse no estudo da aplicação dos polímeros 
condutores em Materiais Absorvedores de Radiação (MAR) se deve, principalmente, à facilidade de preparação desse tipo de polímero e de obtenção de MAR com menor massa específica. O uso de MAR tem aumentado significativamente, podendo-se citar a sua aplicação em televisores, computadores, telefones celulares, salas de instrumentação, antenas de rádio-transmissão, equipamentos de comunicação, entre outras, de modo a se evitar que circuitos eletrônicos sofram interferência de radiação gerada por motores elétricos ou redes de alta tensão, ou para minimizar ou eliminar a presença de radiações danosas ao meio ambiente.

O uso de polímeros condutores na formulação de MAR tem sido realizado por deposição ou mistura em um material suporte (matriz), desejando-se que o polímero condutor permaneça estável durante a utilização do produto final. Para esse fim torna-se grande o interesse em se obter polímeros condutores que apresentem boa estabilidade em condições ambiente, quando utilizados em blendas poliméricas. Yoshino e cols. ${ }^{[1]}$ foram os primeiros autores a reportarem o uso do polipirrol em aplicações eletromagnéticas. Recentemente, muitos outros trabalhos têm sido realizados para mostrar a potencialidade dos polímeros condutores para esta aplicação, Tabela 1.

A faixa de freqüência onde aparecem os maiores problemas de interferência eletromagnética é de 50$60 \mathrm{~Hz}$ (sistema estéreo) a $10^{9}-10^{11} \mathrm{~Hz}$ (transmissão de radar). No entanto, a perturbação mais comum ocorre na frequiência de $10^{4} \mathrm{~Hz}$, historicamente cha-
Tabela 1. Uso dos polímeros condutores intrínsecos em aplicações eletromagnéticas ${ }^{[2-5]}$

\begin{tabular}{lc}
\hline \multicolumn{1}{c}{ Polímeros } & Aplicações \\
\hline Polianilina (PAni) & EMI*, ESD** \\
Polipirrol (filmes) & EMI \\
Polipirrol em matriz poliéster & EMI, MAR \\
Polipirrol em matrizes elastoméricas & MAR \\
Poli(3-octil-tiofeno) nas matrizes & EMI \\
PS, PVC e EVA & EMI, MAR \\
Poli(p-fenileno benzo-bis-tiazol)
\end{tabular}

*EMI: Interferência eletromagnética; **ESD: Dissipação eletrostática

mada de radio-freqüência (RFI), devido ao maior uso em sistemas de comunicação, Figura 1.

\section{Propriedades dielétricas dos polímeros condutores}

Os polímeros condutores são também denominados de "metais sintéticos" por possuírem propriedades elétricas, eletrônicas, magnéticas e ópticas semelhantes às dos metais. Os polímeros condutores passam de isolantes a condutores pela adição de agentes de transferência de carga. Estes são chamados de "dopantes" em analogia aos semicondutores inorgânicos, e o seu uso acarreta em uma dramática mudança nas propriedades dos polímeros mencionadas acima. Controlando a quantidade e o tipo de dopante é possível modelar a condutividade do material.

Um comportamento bastante interessante dos polímeros condutores é a variação da condutividade com a freqüência das ondas incidentes. Por apresen-

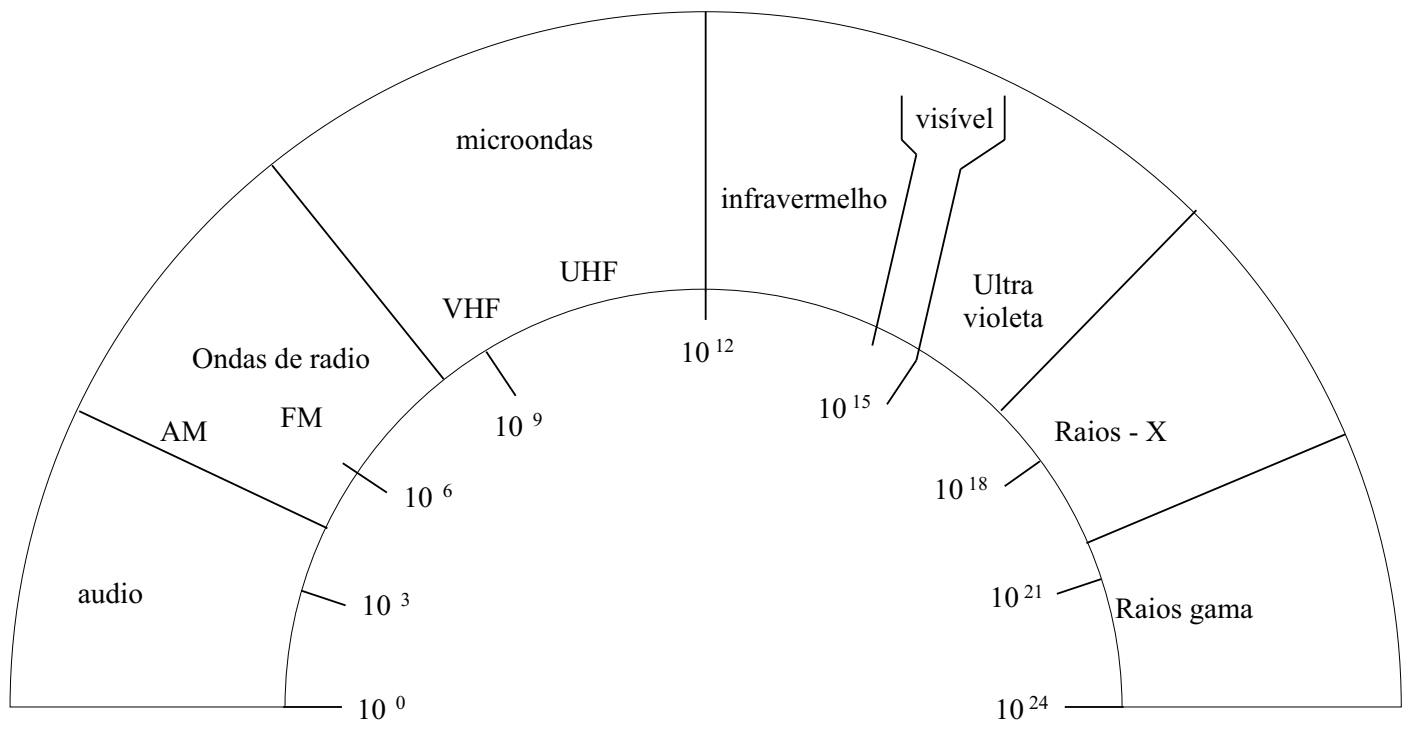

Figura 1. Espectro eletromagnético. Valores de freqüência em Hz. 
tar este comportamento é possível utilizar os polímeros condutores como absorvedores de radiação. O desenvolvimento dos polímeros condutores para esta aplicação implica no conhecimento de algumas características físicas, tais como: permissividade elétrica $(\varepsilon)$ e permeabilidade magnética $(\mu)$ em função da frequiência. Conhecendo-se estes parâmetros físicos é possível inferir sobre as propriedades de absorção, além de se poder variar a formulação do material para obter maiores ou menores valores da constante dielétrica $(\varepsilon)$. O valor de $\varepsilon$ é utilizado para quantificar o comportamento de um material quando este é submetido a um campo eletromagnético. A forma clássica de estudar a relaxação dielétrica dos polímeros condutores é inspirada nos materiais dielétricos.

A principal característica dessa análise é tentar separar a condutividade estática $\left(\sigma_{\mathrm{dc}}\right)$ e a condutividade que aparece após a aplicação de uma dada frequiência $\left(\sigma_{\mathrm{ac}}\right)$. Para uma freqüência angular $\omega(\omega=2 \pi \mathrm{f})$, a condutividade é: $\sigma(\omega)=\sigma_{\mathrm{dc}}+\sigma_{\mathrm{ac}}(\omega)$. Da mesma forma, quando os resultados são expressos em termos de permissividade $\varepsilon^{*}\left(\varepsilon^{*}=\varepsilon^{\prime}-\right.$ i $\left.\varepsilon^{\prime \prime}\right)$, a contribuição dos mecanismos de polarização e condução podem ser separados. O valor de $\varepsilon$ ' está relacionado com a capacidade do material em armazenar energia e $\varepsilon$ " expressa a habilidade do material perder energia como calor. Um fator importante no estudo de materiais com potencialidade para aplicação como absorvedores é o conhecimento da tangente de perda $(\tan \delta=\varepsilon " / \varepsilon$ '). Por exemplo, em um material ideal, homogêneo e isotrópico, a constante dielétrica $\varepsilon$ e a condutividade $\sigma_{\mathrm{dc}}$ são constantes em toda a faixa de frequiência. No entanto, em um material real como os polímeros condutores a condutividade e, portanto, a constante dielétrica, varia com a freqüência. Por exemplo, altos valores de $\varepsilon$ para baixas frequiências estão relacionados com a heterogeneidade dos materiais (a condutividade varia ao longo dos caminhos condutores) criando fenômenos de polarização. A um nível macroscópico Matveeva ${ }^{[6]}$ sugere que a condutividade nos polímeros condutores seja governada pelos transportes intermolecular (ou intercadeia), intramolecular e interpartículas. A dopagem destes polímeros fornece muitos portadores de carga em potencial, que precisam se mover para contribuir com a condutividade. $\mathrm{O}$ fator limitante no processo de condução de um polímero dopado é, portanto, a mobilidade dos portadores. Sendo assim, qualquer mudança estrutural na cadeia polimérica afetará as propriedades condutoras.
Existem muitos trabalhos na literatura sobre a caracterização das propriedades dielétricas dos polímeros condutores na faixa de $1 \mathrm{MHz}$ a $20 \mathrm{GHz}$, devido ao estudo das propriedades de transporte destes materiais ${ }^{[7,8]}$. Em particular, a resposta dielétrica é importante para o entendimento da natureza metálica dos polímeros condutores.

Olmedo e cols. ${ }^{[9]}$ verificaram a influência de alguns parâmetros estruturais, como o tamanho do dopante (contra-íon) e o comprimento da cadeia alifática dos monômeros substituídos, nas propriedades dielétricas. Quando o tamanho do contra-íon aumenta há uma diminuição nos valores de $\varepsilon$ '. Isto é atribuído ao aumento da distância intramolecular o que dificulta o "salto eletrônico" (hopping) entre as cadeias. Desta maneira, as propriedades dielétricas dos polímeros condutores são amplamente dependentes do tipo de síntese e do dopante, que podem causar diferenças na estrutura molecular modificando, consequentemente, as propriedades eletromagnéticas do MAR. Essas características exclusivas dos polímeros condutores os tornam uma classe de centros de absorção moldáveis, permitindo realizar ajustes nas características finais dos materiais absorvedores.

\section{Blindagem eletromagnética}

A blindagem eletromagnética por reflexão ou por absorção é de grande interesse para fins militares e civis. Muitos dispositivos eletrônicos que inerentemente, mas não intencionalmente, transmitem sinais indesejáveis, são envolvidos em caixas condutoras protegendo efetivamente os componentes de fontes externas de radiação, e ao mesmo tempo prevenindo o escape de ruído eletromagnético do próprio componente ${ }^{[10,11]}$. Níveis de condutividade necessários para bons valores de blindagem podem ser alcançados com a inclusão de cargas condutoras (negro de fumo, grafite, carbono vítreo, partículas metálicas ou mistura de óxidos metáli$\cos$ ) em matrizes poliméricas. Essas matrizes aditadas com esses tipos de cargas são chamadas de "polímeros condutores extrínsecos". A natureza abrasiva dos aditivos metálicos e a grande quantidade de negro de fumo necessária para alcançar os valores de condutividade são algumas desvantagens dos polímeros condutores extrínsecos. Desta forma, o desenvolvimento de condutores plásticos baseados nos "polímeros condutores intrínsecos" é muito promissor, pois as limitações citadas acima não estão presentes. A combinação de algumas propriedades como alta condutividade, baixa 
densidade e a facilidade de processamento tornam estes materiais interessantes para o uso como absorvedores de radiação.

A relação entre a efetividade de blindagem (EB) e a resistividade de um material condutor pode ser obtida considerando-se o que ocorre quando uma onda plana atinge o material. Uma parte da radiação é refletida e a outra absorvida, equações 1 e 2 , respectivamente. Uma parte desprezível do sinal é perdida por multi-reflexão interna. A atenuação total da radiação da onda plana (SE) é a soma das contribuições das radiações refletida (r) e absorvida (A), equação 3. Onde: $\rho$ é a resistividade volumétrica da amostra (ohm.cm), $\mu$ é a permeabilidade magnética relativa da amostra (geralmente 1,0), f é a frequiência da radiação (Hz) e L é a espessura da amostra (cm). A Tabela 2 mostra a relação entre a atenuação da radiação (refletividade) e a porcentagem de energia absorvida.

$$
\begin{aligned}
& r=108,2+10 \log (1,7 / \rho \mu f) \\
& A=0,00168 L(\mu f / \rho)^{1 / 2} \\
& S E=r+A
\end{aligned}
$$

Ruckenstein e Park ${ }^{[12]}$ prepararam compósitos à base de polipirrol e obtiveram EB de $26 \mathrm{~dB}$ na faixa de freqüência de 1 a $2 \mathrm{GHz}$. Taka ${ }^{[13]}$ preparou compósitos baseados no polímero condutor politiofeno e obteve cerca de $50 \%$ de EB em relação ao níquel na faixa de $100 \mathrm{MHz}$. Colaneri e Shacklette ${ }^{[14]}$ obtiveram valores de EB de $40 \mathrm{~dB}$ para blendas de PAni e termoplásticos preparadas por processamento reativo. Olmedo e cols. ${ }^{[15]}$ desenvolveram materiais absorvedores de microondas a base de polipirrol, polianilina e poli(3-octil-tiofeno) obtidos por oxidação química. Verificaram que as propriedades de reflexão de blendas de polipirrol / PVC ( $45 \mathrm{~dB}$ em $13 \mathrm{GHz}$ para um material com $2 \mathrm{~mm}$ de espessura) são similares às de um material magnético granular (dispersão de pó de ferrocarbonila em um elastômero), e que a massa superficial da blenda foi de $2,6 \mathrm{Kg} . \mathrm{cm}^{-2}$ comparada com $5 \mathrm{Kg} . \mathrm{cm}^{-2}$ para o material magnético granular. Kathirgamanathan ${ }^{[16]}$ preparou materiais absorvedores de microondas aplicando polímeros condutores como adesivo entre as camadas de termoplásticos. Courric e Tran ${ }^{[17]}$ prepararam filmes de poli(p-fenileno-vinileno) por moldagem utilizando diferentes dopantes e verificaram que a eficiência de blindagem destes materiais depende da natureza do
Tabela 2. Relação entre atenuação da radiação (refletividade) e porcentagem de energia absorvida ${ }^{[19]}$.

\begin{tabular}{cc}
\hline $\begin{array}{c}\text { Atenuação de Refletividade, } \\
\text { dB }\end{array}$ & \% da Energia Absorvida \\
\hline 0 & 0 \\
-3 & 50 \\
-10 & 90 \\
-15 & 96,9 \\
-20 & 99 \\
-30 & 99,9 \\
-40 & 99,99 \\
\hline
\end{tabular}

dopante e da espessura da amostra, obtendo valores de EB de $30 \mathrm{~dB}$ para faixa de frequiência de 3-5 GHz. Verificaram que, apesar de todas as amostras apresentarem valores de condutividade satisfatórios para o uso em absorvedores de radiação $\left(10^{-3}\right.$ a $10^{-1}$ S.cm-1), essas apresentaram diferentes valores de absorção. Este fato foi atribuído ao uso de diferentes dopantes que, apesar de favorecer a obtenção de amostras com valores iguais de condutividade, modifica as propriedades dielétricas do material, bem como, a impedância e portanto, a eficiência de blindagem. Makelä e cols ${ }^{[18]}$ prepararam filmes laminados de PAni dopada com ácido cânfor-sulfônico (CSA) e policarbonato $(1-30 \mu \mathrm{m})$ e obtiveram efetividade de blindagem de $30 \mathrm{~dB}$.

Uma característica importante da PAni é a variação da condutividade, e portanto, da constante dielétrica $\left(\varepsilon^{\prime}\right)$, em função do nível de dopagem, do tipo de dopante e do método de síntese. Sendo assim, a ampla faixa de condutividade e as diferenças na estrutura molecular devido ao uso de diferentes dopantes permite formular absorvedores muito eficientes. Trivedi e cols. ${ }^{[20]}$ prepararam materiais absorvedores de radiação utilizando compósitos baseados em PAni e Nylon. Estudaram diferentes ácidos dopantes (ácido p-tolueno sulfônico (TSA), sulfosalicílico (SSA) e benzeno sulfônico (BSA)) na eficiência de blindagem entre $1 \mathrm{MHz}$ e $1 \mathrm{GHz}$, verificando uma grande dependência nos valores de EB em função do ácido dopante. Para altas frequiências EB foi de 16-18 dB e para baixas frequiências os valores foram maiores que $40 \mathrm{~dB}$.

\section{Preparando materiais absorvedores de radiação}

A faixa de frequiência em que os absorvedores de radiação trabalham é de $10 \mathrm{MHz}$ a $100 \mathrm{GHz}$. A 
absorção ocorre porque estes materiais têm a propriedade de trocar energia eletromagnética por energia térmica. Desta maneira, podem ocorrer relaxações na cadeia do polímero para que ele possa fazer esta conversão de energia. É por esta razão que as propriedades físico-químicas e estruturais dos polímeros condutores têm grande influência na evolução de suas propriedades dielétricas como apresentado anteriormente. Levando-se em consideração a faixa de condutividade específica dos polímeros condutores $\left(1-10^{5} \mathrm{~S} \mathrm{~cm}^{-1}\right)$ é necessário diluir a fase condutora para se obter materiais com condutividade apropriada para uso em absorvedores de radiação. É importante ressaltar que estes polímeros apresentam propriedades mecânicas insatisfatórias e o seu processamento é muito difícil, devido à sua insolubilidade em solventes comuns e à sua infusibilidade. Portanto, ao se preparar materiais absorvedores de radiação é necessário selecionar um material como matriz (polímero isolante, substrato poroso, etc.), que permita a obtenção de um composto com as seguintes características: (i) condutividade modelada; (ii) possibilidade de usar técnicas de processamento de polímeros (extrusão, injeção,...) e (iii) boas propriedades mecânicas.

Os diferentes tipos de polímeros condutores encontrados recentemente na literatura também corroboram para melhorar a manufatura de materiais absorvedores de radiação, devido às especificidades particulares de cada um, por exemplo: polipirrol, pela sua enorme habilidade de ser sintetizado "in situ" nas matrizes isolantes; polianilina, pelas suas características físico-químicas específicas e sua facilidade de síntese.

\section{Tipos de materiais}

Os materiais devem ser escolhidos de acordo com a freqüência utilizada. Duas principais classes de materiais são utilizadas: (i) materiais densos compostos por uma única fase e (ii) materiais compostos por pelo menos duas fases (matriz e carga magnética e/ou dielétrica). A primeira classe é utilizada quando a absorção ocorre em uma larga faixa de frequiência. As propriedades devem ser homogêneas em todo material e, geralmente, são utilizados materiais magnéticos como ferritas. A segunda classe de materiais envolve a dispersão de uma carga com propriedades magnéticas ou dielétricas em uma matriz isolante. A dispersão da carga na matriz é a principal variável, a qual pode alterar as propriedades finais do material, devido às diferen- tes formas de agregação das partículas sendo que, a função das partículas condutoras está associada ao controle de transferência eletrônica inter-partícula. Outro fator muito importante para a obtenção de materiais absorvedores de radiação é a densidade superficial do material. Materiais obtidos pela adição de cargas inorgânicas possuem geralmente altas densidades. Materiais condutores obtidos pela adição de polímeros condutores apresentam densidades relativamente menores. Os métodos de preparação de compostos condutores utilizando polímeros condutores oferecem alta reprodutibilidade, em relação às cargas inorgânicas, 0 que viabiliza o processamento destes materiais.

Materiais absorvedores de radiação geralmente são preparados usando múltiplas camadas com materiais de diferentes propriedades dielétricas e magnéticas, ao invés de usar um único material. Olmedo e cols. ${ }^{[21]}$ relataram recentemente algumas propriedades específicas dos absorvedores preparados com polímeros condutores. Para obter um máximo desempenho, a resistividade das placas deve variar de altos valores na parte frontal a baixos na parte posterior, o que consiste em alterar lentamente a impedância entre os materiais para minimizar as reflexões. A faixa de radiação a ser utilizada é dependente do número de placas utilizadas e das propriedades intrínsecas de cada uma. Sendo assim, há uma grande dificuldade em controlar as propriedades do produto final. Uma das vantagens da preparação de blendas condutoras baseadas em polímeros condutores é a possibilidade de modelar a condutividade do material, pois ao contrário do que ocorre com cargas inorgânicas, o limite de percolação destes materiais é muito abaixo do nível alcançado pelas cargas inorgânicas.

O limite de percolação é definido como a "quantidade mínima de carga necessária para se chegar ao "limiar de aumento de condutividade". Estudos teóricos mostram que, para cargas inorgânicas o "limiar" é alcançado quando se usa $16 \%$ (v/v) de carga ${ }^{[22]}$. Para blendas de polímeros condutores os valores chegam a ser 13 vezes menores ${ }^{[23-25]}$. Isto é explicado pela formação de redes interpenetrantes entre os componentes da mistura. Desta forma, são necessárias pequenas quantidades de polímero condutor para se obter valores de condutividade suficientes para o seu uso em blindagem de interferência eletromagnética - EMI $\left(10^{-2}-10^{2} \mathrm{~S}^{-\mathrm{cm}^{-1}}\right)$ e proteção antiestática- ESD $\left(10^{-11}-10^{-2} \mathrm{~S}^{-1} \mathrm{~cm}^{-1}\right)^{[26]}$, o qual não modifica as propriedades mecânicas e a densidade do polímero isolante. 


\section{Experimental}

\section{Síntese do polímero condutor polianilina}

A PAni foi sintetizada quimicamente em escala prépiloto (reator de vidro com capacidade de $10 \mathrm{~L}$ e encamisado) usando uma solução $3,0 \mathrm{~mol} \times \mathrm{L}^{-1}$ de $\left(\mathrm{NH}_{4}\right)_{2} \mathrm{~S}_{2} \mathrm{O}_{8}$ como oxidante, a qual foi gotejada sobre uma solução contendo 0,8 mol $\times \mathrm{L}^{-1}$ de anilina $(\mathrm{K}=0,8)$. 3,0 mol $\times \mathrm{L}^{-1}$ de $\mathrm{HCl}$ e $3,0 \mathrm{~mol} \times \mathrm{L}^{-1}$ de $\mathrm{NaCl}$. O pó obtido foi filtrado, lavado com água até obtenção de solução incolor ${ }^{[27]}$. A polianilina dopada com $\mathrm{HCl}$ foi desdopada com uma solução de $\mathrm{NH}_{4} \mathrm{OH} 1,0$ mol $\times \mathrm{L}^{-1}$ durante $24 \mathrm{~h}$ para se obter a PAni base esmeraldina na forma de um pó preto. A dopagem da PAni esmeraldina em meio ácido dodecilbenzeno sulfônico (DBSA), razão 1:2, foi realizada por processamento reativo no misturador acessório do Haake Rheocord 90 $0^{[27,28]}$.

\section{Preparação das misturas}

As blendas EPDM/PAni-(DBSA) $)_{2}$ foram preparadas no misturador mecânico interno, acessório do Reômetro Haake Rheocord 90. Preparou-se misturas variando a concentração do centro absorvedor (PAni) de 30 a $80 \%(\mathrm{~m} / \mathrm{m})$. As condições de mistura foram: $150^{\circ} \mathrm{C}, 30 \mathrm{rpm}$ por $15 \mathrm{~min}^{[29]}$. À partir destas amostras foram preparadas mantas de $15 \mathrm{~cm}$ x $15 \mathrm{~cm}$ por 1 e $3 \mathrm{~mm}$ de espessura, utilizando a técnica de moldagem por compressão a $100{ }^{\circ} \mathrm{C}$.

\section{Medidas de refletividade}

Foram realizadas utilizando o método do arco NRL o qual foi concebido no Laboratório de Pesquisa Naval dos Estados Unidos (Naval Research Laboratories), na década de 50. O arco consiste de uma estrutura de madeira que permite fixar um par de antenas em uma variedade de ângulos. A antena, normalmente do tipo corneta, pode ser deslocada ao longo deste arco. A amostra a ser caracterizada é posicionada sobre um pequeno pedestal no centro da curvatura do arco. A estrutura do arco é projetada de modo a manter a corneta apontada para o centro do corpo-de-prova em teste. $\mathrm{O}$ transmissor e o receptor podem ficar próximos, contanto que, seja colocado um material absorvedor entre eles, de modo a reduzir as interferências da corneta transmissora e da corneta receptora entre $\mathrm{si}^{[30]}$. As medidas de refletividade foram realizadas na faixa de frequência de $8-12 \mathrm{GHz}$ na temperatura ambiente.

\section{Resultados e Discussão}

As Figuras 2 (a-b) e 3 (a-b) mostram as curvas de refletividade de blendas de EPDM/PAni-(DBSA) $)_{2} \mathrm{em}$ função da frequiência e da concentração do polímero condutor para mantas de $1 \mathrm{~mm}$ de espessura. Para amostra contendo $30 \%$ PAni-(DBSA) $)_{2}$ verifica-se na faixa de frequiência 9-10 GHz uma atenuação média de $50 \%$ da radiação eletromagnética incidente . Para as blendas contendo 40 e $50 \%$ na faixa de frequiência 10,5-12 GHz observa-se uma atenuação de $-4 \mathrm{~dB}$ (aproximadamente $60 \%$ da radiação é absorvida). Nestas concentrações pode-se dizer que este material tem comportamento ressonante, ou seja absorve a radiação em uma faixa estreita de radiação ${ }^{[19]}$. Para amostras com concentrações acima de 50\% de PAni$(\mathrm{DBSA})_{2}$ verifica-se um comportamento diferente, ou seja, o material passa a ter um comportamento de banda larga (absorve em toda a faixa de frequiência estudada $)^{[19]}$.

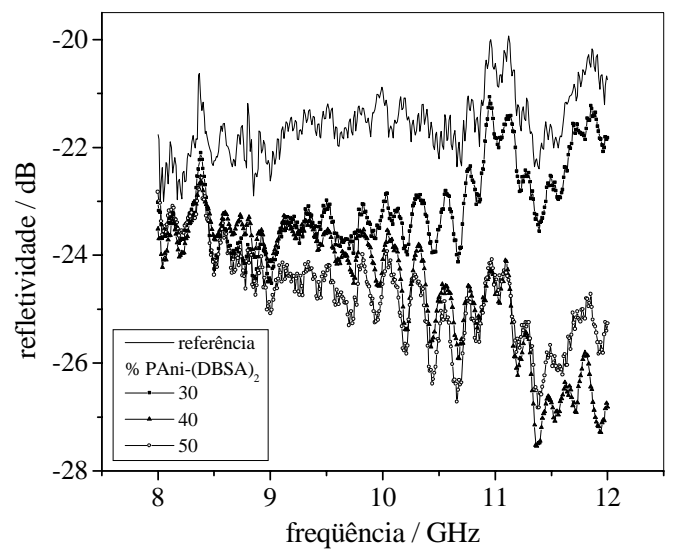

(a)

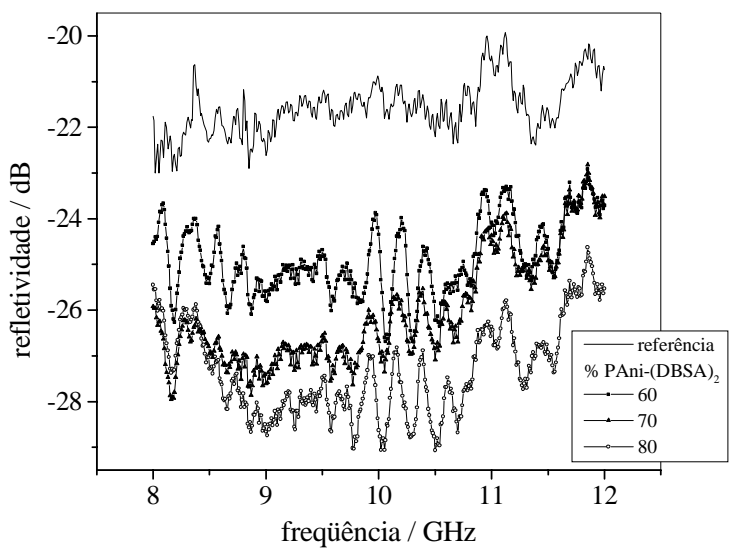

(b)

Figura 2. Curvas de refletividade na faixa de $8-12 \mathrm{GHz}$ para blendas de EPDM/PAni-DBSA para mantas com espessura de (a) $1 \mathrm{~mm}$ e (b) $3 \mathrm{~mm}$ 
Este resultado é bastante interessante, pois a literatura considera os polímeros condutores como absorvedor de banda estreita de freqüências ${ }^{[9,15,17,21]}$. O comportamento de absorvedor tipo banda larga observado nas blendas preparadas deve-se, provavelmente, à dispersão do peso molecular da polianilina, onde as cadeias de vários tamanhos apresentam diferentes valores de condutividade, formando um composto com condutividade microscópica variável (a condutividade varia ao longo dos caminhos condutores). Como a absorção da radiação eletromagnética em uma determinada freqüência está relacionada com a condutividade, a contribuição destes diferentes valores confere à blenda obtida a característica de absorvedor tipo banda larga. Desta forma, blendas contendo 60, 70 e $80 \%(\mathrm{~m} / \mathrm{m})$ de PAni-(DBSA) $)_{2}$ absorvem em média 60,75 e $80 \%$ da radiação na faixa de $8-12 \mathrm{GHz}$, respectivamente. Aumentando-se a espessura das mantas para $3 \mathrm{~mm}$, nas concentrações de $30-50 \%$, verifica-se um comportamento similar ao

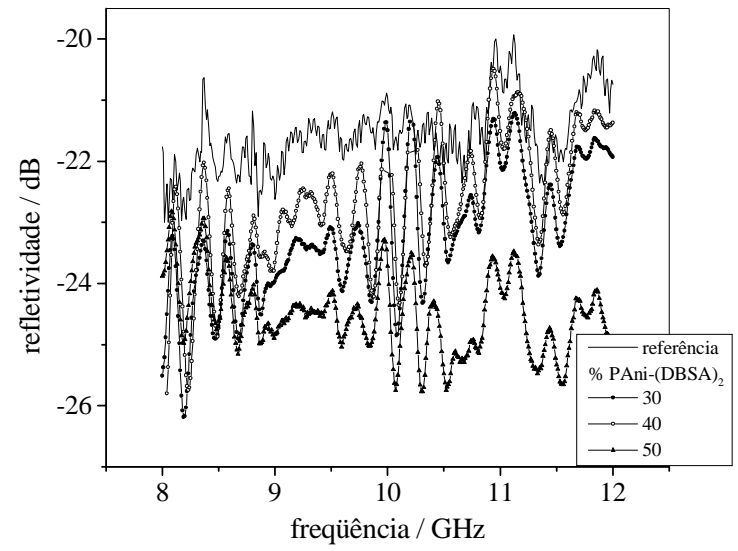

(a)

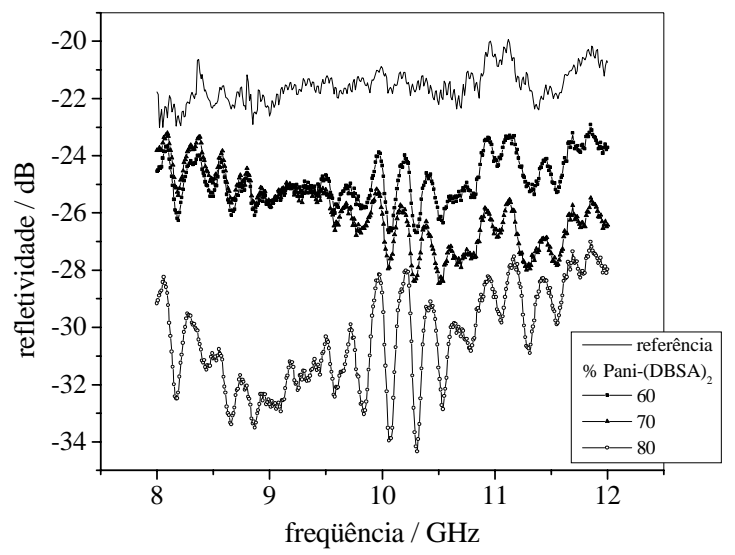

(b)

Figura 3. Curvas de refletividade na faixa de $8-12 \mathrm{GHz}$ para blendas de EPDM/PAni-(DBSA) ${ }_{2}$ para mantas com espessura de (a) $1 \mathrm{~mm} \mathrm{e} \mathrm{(b)} 3 \mathrm{~mm}$ observado para as amostras com $1 \mathrm{~mm}$ de espessura. Para as concentrações de 70 e $80 \%$ do polímero condutor foram obtidos melhores valores de atenuação da radiação incidente, correspondendo a 75 e $90 \%$ de absorção, respectivamente.

\section{Conclusão}

O desenvolvimento dos polímeros condutores, desde 1977, tem sido dedicado principalmente aos fundamentos físicos e químicos para melhor compreender as propriedades destes materiais. Dentro deste contexto, o comportamento destes tipos de polímeros, quando submetidos a campos eletromagnéticos na faixa de frequiência de microondas, têm sido estudado para o entendimento das propriedades de transporte, principalmente em função da freqüência e da temperatura. Estudos paralelos têm sido realizados para a aplicação destes materiais em áreas ligadas diretamente com a condutividade, como absorvedores de radiação. Porém, o principal fator que coloca os polímeros condutores em um lugar privilegiado neste tipo de aplicação está relacionado com a diversidade de caminhos que podem ser utilizados para a preparação destes materiais. $\mathrm{O}$ trabalho que vem sendo realizado, embora preliminar, usando blendas de EPDM/PAni-DBSA, com amostras de diferentes espessuras, mostra atenuação da radiação incidente na faixa de 4-10 dB, ou seja, de aproximadamente 50 a $90 \%$ de absorção, na faixa de frequiência de 8-12 GHz. Isto indica que a blenda em estudo é um material promissor em aplicações de blindagem eletromagnética. Outras variáveis estão sendo avaliadas como tempo de processamento da mistura e razão PAni/ácido.

\section{Agradecimentos}

Os autores agradecem à FAPESP pelo apoio financeiro (Proc. No. 99/03347-2) e Oscar Kasuo Kosima e Dermeval Carinhana Júnior pelo apoio na síntese da polianilina.

\section{Referências bibliográficas}

1. Yoshino, K.; Tabat, M.; Kaneto, K. \& Ohhsawa, T. - J. Appl. Phys., 24, p.693 (1985).

2. Joo, J. \& Epstein, A. - J. Apply. Phys. Lett., 65, p.2278 (1994). 
3. Javadi, H.H.S.; Cromack, K.R.; MacDiarmid, A.G. \& Epstein, A.J. - J. Phys. Rev. B, , 39, p.3579 (1989).

4. Kuhn, H.H.; Child, A.D. \& Kimbrell, W.C. - Synth. Met., 71, p.2139 (1995).

5. Kaynak, A.; Unsworth, J.; Clout, R.; Mohan, A.S. \& Beard, G.E. - J. Appl. Poly. Sci., 54, p.269 (1994).

6. Matveeva, E.S. - Synth. Met., 79, p.127 (1996).

7. Pinto, N.J.; Sinha, G.P. \& Aliev, F.M. - Synth. Met., 94, p.199 (1998).

8. Singh, R.; Arora, V.; Tandon, R.P.; Mansingh, A. \& Chandra, S. - Synth. Met., 104, p.137 (1999).

9. Houquerbie, P \& Olmedo, L. - Synth. Met., 65, p.19 (1994).

10. Oberholtzer, L.C.; in "Encyclopedia of Polymer Science and Engineering”, Mark, H.F., Bicales, N.M., Overberger, C.G., Menges, G., Kroschwitz, J.I., eds., John Wiley \& Sons, $2^{a}$ ed., Nova Iorque, vol. 5, p. 609 (1987).

11. Simon, R.M. in "Encyclopedia of Polymer Science and Engineering", Mark, H.F., Bicales, N.M., Overberger, C.G., Menges, G., Kroschwitz, J.I.,

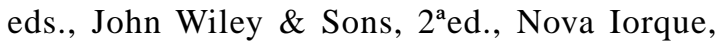
Supplement, p. 155 (1987).

12. Ruckenstein, E. \& Park, J.S. - Polym. Compos., 12, p.289 (1981).

13. Taka, T. - Synth. Met., 41-43, p.1177 (1991).

14. Colaneri, N. \& Shacklette, L. - IEEE Trans. Instrum. Meas., 41, p.291 (1992).

15. Olmedo, L.; Hourquebie, P. \& Jousse, F. - Adv. Mater., 5, p.373 (1993).

16. Kathirgamanathan, P. - Polymer, 34, p.3105 (1993).

17. Corric, S. \& Tran, V.H. - Polymer, 39, p.2399 (1999).
18. Makelä, T., Pienimaa, S., Taka, T., Jussila, S. \& Isotalo, H. - Synth. Met., 85, p.1355 (1997).

19. International Encyclopedia of Composites, vol. 6, VHC Publishers, New York, (1991).

20. Trivedi, D.C. \& Dhawan, S.K. - Synth. Met., 59, p.267 (1993).

21. Olmedo, L.; Houquerbie, P e Jousse, F., in "Handbook of Organic Conductive Molecules and Polymers”, ed. H.S. Nalwa, John Wiley, Vol.3, (1997)

22. Zallen, R. - "The Physics of Amorphous Solids", John Wiley \& Sons, New York, (1983).

23. Faez, R.; Gazotti, W.A \& De Paoli, M.-A. - Polymer, 40, p.5497 (1999).

24. Banerjee, P \& B.M Mandal,. - Synth.Met., 74, p.257 (1995).

25. Gazotti, W.A., Faez, R. e De Paoli, M.-A. - Eur. Polym. J., 35, p.35 (1999).

26. Catálogo da RTP Immagineering Plastics Company

27. Patrícia S. de Freitas, "Preparação de polianilina em escala piloto e seu processamento" Tese de doutorado (em andamento), UNICAMP, Campinas.

28. Titelman, G.I., Zilberman, M., Siegmann, A., Haba, Y., Narkis, M. - J. Appl. Polymer Sci., 66, p. 2199, 1997.

29. Faez, R. \& De Paoli, M.-A. - Eur. Poly. J., submetido

30. Josiane de Castro Dias "Revestimentos de materiais absorvedores de radiação (2-18 GHz) com aplicação no setor aeronáutico e telecomunicações" Tese de Doutorado, ITA, Junho de 2000.

Recebido: $22 / 02 / 00$ Aprovado: 24/08/00 